
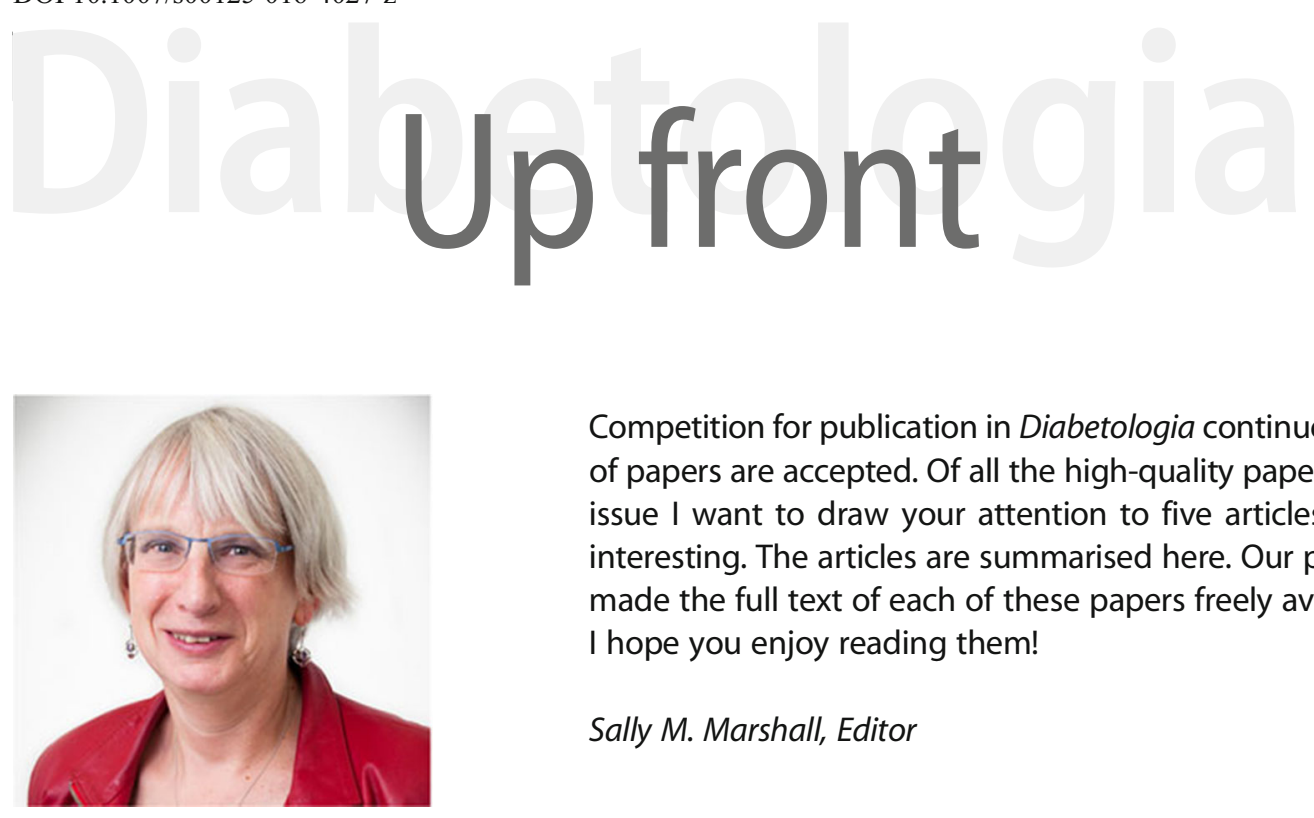

Competition for publication in Diabetologia continues to grow, and less than 20\% of papers are accepted. Of all the high-quality papers that appear in this month's issue I want to draw your attention to five articles that I think are particularly interesting. The articles are summarised here. Our publisher, Springer, has kindly made the full text of each of these papers freely available.

I hope you enjoy reading them!

Sally M. Marshall, Editor

\section{Islet biology, the CDKN2A/B locus and type 2 diabetes risk}

Yahui Kong, Rohit B. Sharma, Benjamin U. Nwosu, Laura C. Alonso

Genome wide association studies have identified a range of genomic loci that influence type 2 diabetes risk in human populations. Despite this advance, the mechanistic pathways linking polymorphisms to disease occurrence mostly remain unclear. Even for $C D K N 2 A / B$, a locus that has been intensely scrutinised and has relevance to cancers and ageing as well as diabetes, diabetes risk mechanisms remain poorly understood. In this issue, Kong et al review the current understanding of how $C D K N 2 A / B$ polymorphisms affect the function of diabetes-relevant tissues. In particular, this review includes an in-depth look at islet biology, which is widely assumed to be the site of action of $C D K N 2 A / B$ polymorphisms since a $C D K N 2 A / B$ locus gene product, p16, regulates beta cell proliferation. In combining literature relating to clinical phenotyping with basic scientific observations, Kong et al challenge the notion that $C D K N 2 A / B$ polymorphisms act entirely through their effects on beta cell biology.
Insulin pump basal adjustment for exercise in type 1 diabetes: a randomised crossover study

Sybil A. McAuley, Jodie C. Horsburgh, Glenn M. Ward, André La Gerche, Judith L. Gooley, Alicia J. Jenkins, Richard J. Maclsaac, David N. O'Neal

Maintaining normoglycaemia during and after exercise remains a challenge for many with type 1 diabetes. Clinical guidelines recommend halving insulin pump basal delivery $1 \mathrm{~h}$ pre-exercise to prevent hypoglycaemia. However, these guidelines are derived mainly from expert opinion in the absence of scientific evidence. In this issue, McAuley et al report that, when subcutaneous insulin pump basal delivery is halved $1 \mathrm{~h}$ pre-exercise, there is no significant reduction in circulating insulin levels by the time exercise commences. Subsequently, during $30 \mathrm{~min}$ of moderate-intensity aerobic exercise, a transient increase in circulating insulin occurs. The authors suggest this rise may relate to enhanced insulin absorption due to increased cutaneous blood flow. They also show that hypoglycaemia risk is higher for those with low-normal pre-exercise glucose. To prevent exercise-induced hypoglycaemia, the authors propose that basal reductions in insulin of more 
than $50 \%$ are instituted pre-exercise and that, if commencing exercise with low-normal glucose levels, supplemental carbohydrate should be considered. This article is the subject of a commentary in this issue by Thabit and Leelarathna.

\section{Twenty year fitness trends in young adults and incidence of prediabetes and diabetes: the CARDIA study}

Lisa S. Chow, Andrew O. Odegaard, Tyler A. Bosch, Anne E. Bantle, Qi Wang, John Hughes, Mercedes Carnethon, Katherine H. Ingram, Nefertiti Durant, Cora E. Lewis, Justin Ryder, Christina M. Shay, Aaron S. Kelly, Pamela J. Schreiner

It is generally presumed that higher fitness is associated with lower rates of prediabetes (defined as impaired fasting glucose and/or impaired glucose tolerance) and diabetes development. In this issue, Chow et al use data from the Coronary Artery Risk Development in Young Adults (CARDIA) study $(N=4373)$ to analyse objectively and rigorously the link between cardiorespiratory fitness and development of either prediabetes or diabetes over a 20 year period. The main finding demonstrates that higher cardiorespiratory fitness is associated with lower risk for developing prediabetes and diabetes, even after adjusting for changes in BMI. Since this benefit remained significant even when adjusting for BMI, this study provides evidence that exercise programmes remain critically important for reducing the development of prediabetes and diabetes.

\section{Parenthood and the risk of diabetes in men and women: a 7 year prospective study of $\mathbf{0 . 5}$ million individuals}

Sanne A. E. Peters, Ling Yang, Yu Guo, Yiping Chen, Zheng Bian, lona Y. Millwood, Fiona Bragg, Xue Zhou, Pengfei Ge, Biyun Chen, Yulian Gao, Yijun Li, Junshi Chen, Liming Li, Mark Woodward, Zhengming Chen, on behalf of the China Kadoorie Biobank Collaboration Group

In women, higher parity has been associated with an increased risk of diabetes. It is unclear whether the metabolic consequences of pregnancy and childbearing or socioeconomic and lifestyle factors associated with childrearing underpin this association. In this issue, Peters et al report the results of a large prospective study in which they assessed the association between number of children and diabetes risk separately among men and women. They report that both men and women without children or with multiple children had a higher risk of diabetes compared with those with one child. Each additional child increased the risk of diabetes by $3-4 \%$ in both sexes. This similarity in the relationship between the number of children and diabetes in both women and men suggests that parenthood may increase the risk of diabetes in women with larger families through factors associated with childrearing, rather than through the biological effects of pregnancy and childbearing.

\section{Morphology of the pancreas in type 2 diabetes: effect of weight loss with or without normalisation of insulin secretory capacity}

Ahmad Al-Mrabeh, Kieren G. Hollingsworth, Sarah Steven, Roy Taylor

In type 2 diabetes, the pancreas is recognised both as being smaller than normal and as having a characteristically irregular border. This leads us to ask: are individuals who are born with small pancreases at higher risk of developing type 2 diabetes, or does the pancreas shrink as the condition develops and progresses? Is it possible that smaller pancreas size in type 2 diabetes may be due to the loss of the trophic effects of surges in tissue insulin concentration after meals? In this issue, Al-Mrabeh et al attempt to answer these questions by studying individuals with a wide range of duration of type 2 diabetes before and after substantial weight loss. Increasing duration of diabetes was associated with a more irregular pancreas border. In individuals in whom insulin secretion returned to normal after a very low calorie diet, pancreas volume was higher and border irregularity was reduced compared with those who were unable to resume normal insulin secretory function (typically those with longer duration diabetes). These findings demonstrate that the morphology of the pancreas may provide important prognostic information in type 2 diabetes.

All text supplied by the authors. 